\title{
DNA ligase IV mutations confer shorter lifespan and increased sensitivity to nutrient stress in Drosophila melanogaster
}

\author{
Rashmi Joshi $^{1} \cdot$ Surya Jyoti Banerjee ${ }^{2} \cdot$ Jennifer Curtiss $^{2} \cdot$ Amanda K. Ashley $^{1}[$
}

Received: 14 May 2020 / Revised: 9 March 2021 / Accepted: 4 May 2021 / Published online: 24 November 2021

(c) The Author(s) 2021

\begin{abstract}
The nonhomologous end-joining pathway is a primary DNA double-strand break repair pathway in eukaryotes. DNA ligase IV (Lig4) catalyzes the final step of DNA end ligation in this pathway. Partial loss of Lig4 in mammals causes Lig4 syndrome, while complete loss is embryonically lethal. DNA ligase 4 (DNAlig4) null Drosophila melanogaster is viable, but sensitive to ionizing radiation during early development. We proposed to explore if DNAlig4 loss induced other long-term sensitivities and defects in D. melanogaster. We demonstrated that DNAlig4 mutant strains had decreased lifespan and lower resistance to nutrient deprivation, indicating Lig4 is required for maintaining health and longevity in D. melanogaster.
\end{abstract}

Keywords Drosophila melanogaster $\cdot$ DNA ligase IV $\cdot$ Lifespan $\cdot$ Starvation

\section{Introduction}

DNA damage is a major hallmark of cancer (Hanahan and Weinberg (2011)), and aberrations in pathways maintaining genomic fidelity are associated with multiple cancers (Brown 2017). In mammals, DNA double-strand breaks (DSB) are primarily repaired by homologous recombination (HR) and classical nonhomologous end-joining (cNHEJ); if cNHEJ is compromised, cells may use alternative NHEJ (altNHEJ). Double-strand breaks are recognized by the Ku70-Ku80 dimer, which recruits DNA-PKcs, Artemis, and DNA ligase IV (Lig4) with XRCC4 (Lieber 2010). Lig4 is an ATP-dependent ligase that catalyzes the phosphodiester bond formation in cNHEJ-mediated DSB repair (Lieber 2010), and, in contrast to DNA ligases I and III which facilitate homeostatic DNA metabolism, the activity of Lig4 is restricted to cNHEJ (Lieber 2010).

Drosophila. melanogaster has been extensively used as a model to study DNA repair. The DSB repair in $D$.

Communicated by: Maciej Szydlowski

Amanda K. Ashley

ashleyak@nmsu.edu

1 Department of Chemistry and Biochemistry, New Mexico State University, NM, Las Cruces, USA

2 Department of Biology, New Mexico State University, NM, Las Cruces, USA melanogaster, like in mammals, is via the two primary pathways: the template-dependent homologous recombination and template-independent end-joining (EJ) pathway as well as the alternative end-joining (altEJ) pathway (Gorski 2003; Mota 2019). These pathways and corresponding components in D. melanogaster are similar to the mammalian system, yet there are some key differences. In the D. melanogaster EJ pathway, DSB are recognized by orthologs of the $\mathrm{Ku}$ 70/80 heterodimer: Irbp and Ku80. The D. melanogaster ligation complex is composed of D. melanogaster DNAlig4 and orthologs of XRCC4 (CG3448) and XLF (CG12728 and CG32756). One striking deviation of the D. melanogaster $\mathrm{EJ}$ is the absence of the key protein, DNA-PKcs, a critical component of the mammalian NHEJ pathway (Mota 2019). D. melanogaster EJ pathway also lacks polymerases $\mu$ and $\lambda$ and the nuclease Artemis (recently reviewed (Sekelsky 2017)).

Lig4 hypomorphic mutations in humans cause the Lig4 syndrome (recently reviewed (Altmann and Gennery 2016)), and $\mathrm{Lig}^{-/-}$mice are inviable, as mutations cause p53-mediated neuronal apoptosis resulting in embryonic lethality (Frank 2000). In contrast, D. melanogaster males and females lacking DNAlig4 function are viable and fertile; however, these mutants are hypersensitive to ionizing radiation (IR)-induced DNA damage during early development (Gorski 2003; McVey et al. 2004). An accumulation of DNA damage is commonly observed as a result of aging, and metabolic alterations resulting from nutrient deprivation 
can induce oxidative stress which in turn induces DNA damage (Filomeni et al. 2015). We wanted to investigate whether mutations in DNAlig4 modulates D. melanogaster longevity and response to nutrient deprivation.

\section{Results and discussion}

We obtained three strains of $D$. melanogaster from the Bloomington Drosophila Stock Center: $w^{1118}$ (wild-type DNAlig4) and two DNAlig4 mutants: DNAlig4 $4^{5}$ and DNAlig $4^{57}$ (Gorski 2003). The D. melanogaster stocks were maintained at $25^{\circ} \mathrm{C}$ on normal yeast-cornmeal food (ingredients: $337.5 \mathrm{~g}$ yeast, $195 \mathrm{~g}$ soy flour, $1425 \mathrm{~g}$ cornmeal, $95 \mathrm{~g}$ Drosophila food-grade Agar type II, $900 \mathrm{~g}$ malt extract, $1.5 \mathrm{~g}$ molasses, $100 \mathrm{~mL}$ propionic acid, $250 \mathrm{~mL} \mathrm{10 \%} \mathrm{Tegosept,}$ and $25 \mathrm{~L}$ tap water) unless mentioned otherwise. Flies used for all the experiments were obtained from fertilized eggs laid by homozygous mothers. No differences were observed in fertility or differential distribution of offspring sexes (data not shown).

We confirmed that DNAlig4 mutant strains do not produce DNAlig4 transcripts using qPCR. We designed DNAlig4 primers that recognize the first two exons (completely deleted in the mutant strains). Ten flies of each gender per strain were used for RNA isolation. After homogenization, RNA isolation was accomplished using the trizol-chloroform-ethanol method (Sigma-Aldrich, St. Louis, MO). We used standard conditions for qPCR (detailed here (Joshi et al. 2019)), except that the annealing temperature was adjusted to $60{ }^{\circ} \mathrm{C}$. Primer sets were designed to recognize full-length DNAlig4 transcript. DNAlig4 mRNA expression was assessed by normalizing to Rp49 (Ribosomal protein
L32 (RpL32)). Primer sequences used are as follows: DNAlig4 (full-length DNAlig4 mRNA): 5' GGACACTGGTCG GGATACCT 3', 5'CTGATGGCATCGCTGGAGTC 3'; and Rp49: 5' CCAAGCACTGCATCCGCCACC 3', 5' GCGGGT GCGCTTGTTCGATCC 3'. DNAlig4 Cq values were normalized to Cq values of $R p 49$ via the $2^{-\Delta \Delta \mathrm{Cq}}$ method (Livak and Schmittgen 2001) and analyzed via one-way ANOVA with an ad hoc Dunnett's multiple comparisons test using the statistical software GraphPad Prism. We observed that $\mathrm{Cq}$ values for the housekeeping gene for the three strains fell within one cycle of each other. The relative expression of DNAlig4 in $w^{1118}$ was 1.004 , while in DNAlig $4^{5}$ and DNAlig $4^{57}$, mutant strain expression was 0.0008 or 0.0009 , respectively (Fig. 1A), validating the DNAlig4 mutations result in no Lig4 transcripts.

Next, we evaluated the impact of DNAlig4 loss on lifespan. Adult male and female flies were mated, and embryos were harvested. Per genotype, 100 embryos were collected using forceps and a dissecting microscope and placed into vials containing approximately $10 \mathrm{~mL}$ of standard fly food and stored at $25{ }^{\circ} \mathrm{C}$ for 10 days until eclosion. Freshly emerged male and female virgin flies were collected and stored in groups of 10 , into vials containing approximately $10 \mathrm{~mL}$ of standard fly food. Flies were routinely transferred to fresh vials using $\mathrm{CO}_{2}$ to anesthetize during transfer. The number of dead flies was recorded daily until all flies were dead. A total of 40 flies per strain were assessed. Kaplan-Meier survival curves were created for survivorship using GraphPad Prism, and statistical significance was calculated using the Mantel-Cox log rank test. We observed that $w^{1118}$ flies survived the longest with a median survival of 65.5 days (Table 1). Both the DNAlig4 mutants had significantly shorter lifespans: median survival for mutant

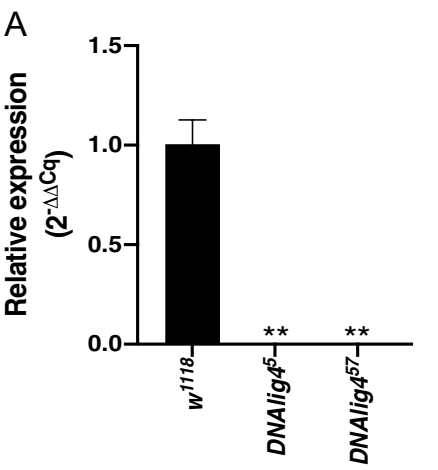

B

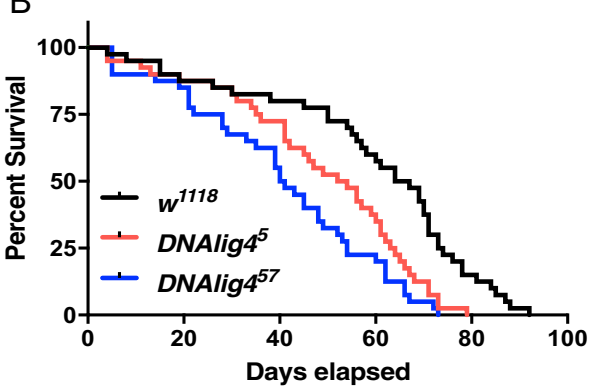

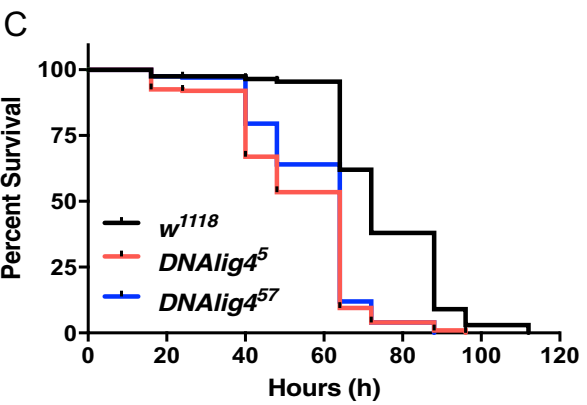

Fig. 1 DNAlig4 mutants have a reduced lifespan and are more sensitive to starvation compared to wild-type $D$. melanogaster. A Transcript levels of DNAlig4 were analyzed by qPCR using primers spanning the first two exons which were deleted in the mutant strains. RNA was harvested from two groups of 10 flies each of mixed sexes. The RNA expression for each of the DNAlig4 mutants was each compared to the wild-type $\left(w^{1118}\right)$ flies. ${ }^{* *} p<0.01, n=2$. B Lifespan was assessed in wild-type $\left(w^{1118}\right)$ and mutant DNAlig4 flies. Median lifes- pan (expressed as days post emergence) is listed in Table 1 ( $p=0.001$ for $w^{1118}$ compared to DNAlig $4^{5}, p<0.0001$ for $w^{1118}$ compared to DNAlig $4^{57}, n=40$ flies per strain). C Survival under starvation conditions was assessed as mentioned in the Results and discussion section. Median survival (expressed as hours into starvation) is listed in Table $1\left(p<0.001\right.$ for $w^{1118}$ compared to DNAlig $4^{5}, p<0.001$ for $w^{1118}$ compared to and DNAlig $4^{57}, n=100$ flies per strain) 
Table 1 Median lifespan of DNAlig4 wild-type and mutant flies and median survival under conditions of nutrient deprivation. Survival curves were obtained using GraphPad Prism; $p$ values were calculated using Mantel-Cox test

\begin{tabular}{llllll}
\hline & $\mathrm{w}^{1118}$ & DNAlig4 $^{5}$ & $p$ value versus $\mathrm{w}^{1118}$ & DNAlig4 $^{57}$ & $p{\text { value versus } \mathrm{w}^{1118}}$ \\
\hline Median lifespan & $65.5 \mathrm{~d}$ & $53 \mathrm{~d}$ & $p=0.001$ & $40.5 \mathrm{~d}$ & $p<0.0001$ \\
Median survival & $72 \mathrm{~h}$ & $64 \mathrm{~h}$ & $p<0.001$ & $64 \mathrm{~h}$ & $p<0.001$ \\
\hline
\end{tabular}

flies was 53 days and 40.5 days for the DNAlig $4^{5}$ and DNAlig $4^{57}$ strains, respectively, and both DNALig4 mutant fly strains were statistically similar to one another (Fig. 1B and Table 1). Our results are in agreement with a previous study where a different DNAlig4 mutant, the DNAlig4 ${ }^{169 a}$, was shown to have reduced lifespan compared to the wild-type strain (Garcia 2011).

We investigated the effect of nutrient deprivation on the survival of DNAlig4 mutants. Briefly, age-matched flies were mated as above, and freshly emerged male and female virgin flies were collected and cultured in groups of 10 into vials containing approximately $10 \mathrm{~mL}$ of standard fly food for $48 \mathrm{~h}$. Flies were then transferred to vials with $1 \%$ agar with water. Dead flies were scored twice a day (in a cycle of $16 \mathrm{~h}$ and $8 \mathrm{~h}$ intervals) until all the flies died; 100 flies per strain were assessed. Survival curves were created for survivorship and statistically analyzed as above. $w^{1118}$ flies were significantly more resistant to nutrient deprivation than either of the DNAlig4 mutant strains, which, again, were statistically similar to one another (Fig. 1C and Table 1).

The reduced lifespan we observed in DNAlig4 mutants is likely a function of accumulation of unresolved DNA damage. We attribute the diminished capacity to withstand nutrient stress to induction of oxidative DNA damage that is resolved with less efficiency in DNAlig4 mutant strains. Overall, we can conclude that while loss of DNAlig4 does not hinder viability of progeny from homozygous mutant parents or manifest any obvious phenotypic defects in $D$. melanogaster, it does negatively impact the lifespan of adult flies and sensitizes them to nutrient deprivation. We conclude that DNA Lig4 is required for maintaining health and longevity in D. melanogaster; the role of DNA Lig4 in supporting health and lifespan of other organisms is currently unknown.

Acknowledgements The authors thank Prisila Ramirez, Michael Burnett, and Philip Yost for assistance with maintaining the fly colonies and breeding.

Author contribution AKA, JC, and RJ designed the experiments. RJ and $\mathrm{SB}$ conducted the experiments, and RJ, SB, and AKA analyzed the data under the supervision of JC and AKA. RJ, SB, JC, and AKA wrote the manuscript.

Funding The research reported in this publication was supported by an Institutional Development Award (IDeA) from the National Institute of General Medical Sciences of the National Institutes of Health under grant number P20GM103451 and by Cowboys for Cancer Research Pilot Grant, all awarded to AKA.

\section{Declarations}

Ethical approval This article does not contain any studies with human participants or vertebrate animals performed by any of the authors.

Conflict of interest The authors declare no competing interests.

Open Access This article is licensed under a Creative Commons Attribution 4.0 International License, which permits use, sharing, adaptation, distribution and reproduction in any medium or format, as long as you give appropriate credit to the original author(s) and the source, provide a link to the Creative Commons licence, and indicate if changes were made. The images or other third party material in this article are included in the article's Creative Commons licence, unless indicated otherwise in a credit line to the material. If material is not included in the article's Creative Commons licence and your intended use is not permitted by statutory regulation or exceeds the permitted use, you will need to obtain permission directly from the copyright holder. To view a copy of this licence, visit http://creativecommons.org/licenses/by/4.0/.

\section{References}

Altmann T, Gennery AR (2016) DNA ligase IV syndrome; a review. Orphanet J Rare Dis 11(1):137

Brown JS et al (2017) Targeting DNA repair in cancer: beyond PARP inhibitors. Cancer Discov 7(1):20-37

Filomeni G, De Zio D, Cecconi F (2015) Oxidative stress and autophagy: the clash between damage and metabolic needs. Cell Death Differ 22(3):377-388

Frank KM et al (2000) DNA ligase IV deficiency in mice leads to defective neurogenesis and embryonic lethality via the $\mathrm{p} 53$ pathway. Mol Cell 5(6):993-1002

Garcia AM et al (2011) Loss of the bloom syndrome helicase increases DNA ligase 4-independent genome rearrangements and tumorigenesis in aging Drosophila. Genome Biol 12(12):R121

Gorski MM et al (2003) The Drosophila melanogaster DNA ligase IV gene plays a crucial role in the repair of radiation-induced DNA double-strand breaks and acts synergistically with Rad54. Genetics 165(4):1929-1941

Hanahan D, Weinberg RA (2011) Hallmarks of cancer: the next generation. Cell 144(5):646-674

Joshi RR, Ali SI, Ashley AK (2019) DNA ligase IV prevents replication fork stalling and promotes cellular proliferation in triple negative breast cancer. J Nucleic Acids 2019:9170341. https:// doi.org/10.1155/2019/9170341

Lieber MR (2010) The mechanism of double-strand DNA break repair by the nonhomologous DNA end-joining pathway. Annu Rev Biochem 79:181-211 
Livak KJ, Schmittgen TD (2001) Analysis of relative gene expression data using real-time quantitative PCR and the $2^{-\Delta \Delta C T}$ method. Methods 25(4):402-408

McVey M, Radut D, Sekelsky JJ (2004) End-joining repair of doublestrand breaks in Drosophila melanogaster is largely DNA ligase IV independent. Genetics 168(4):2067-2076

Mota MBS et al (2019) DNA damage response and repair in perspective: Aedes aegypti, Drosophila melanogaster and Homo sapiens. Parasit Vectors 12(1):533
Sekelsky J (2017) DNA repair in Drosophila: mutagens, models, and missing genes. Genetics 205(2):471-490

Publisher's note Springer Nature remains neutral with regard to jurisdictional claims in published maps and institutional affiliations. 